\title{
SCHRÖDINGER EQUATIONS WITH ASYMPTOTICALLY PERIODIC TERMS
}

\author{
MARCHI, REINALDO DE \\ DEPARTAMENTO DE MATEMÁTICA, UNIVERSIDADE FEDERAL DE MATO \\ GROSSO,CUIABÁ 78060-900, MT, BRAZIL (REINALDODEMARCHI@UFMT.BR)
}

\begin{abstract}
We study the existence of nontrivial solutions for a class of asymptotically periodic semilinear Schrödinger equations in $\mathbb{R}^{N}$. By combining variational methods and the concentration-compactness principle we obtain a nontrivial solution for asymptotically periodic problem and a ground state solution for the periodic problem. In the proofs we apply the Mountain Pass Theorem and its local version.
\end{abstract}

\section{INTRODUCTION}

In this article, we study the existence of nontrivial solutions for the semilinear Schrödinger equation

$$
-\Delta u+V(x) u=f(x, u), x \in \mathbb{R}^{N},
$$

where $V: \mathbb{R}^{N} \rightarrow \mathbb{R}$ and $f: \mathbb{R}^{N} \times \mathbb{R} \rightarrow \mathbb{R}$ are continuous functions. In our main result we establish the existence of solution for the problem (1.1) under an asymptotic periodicity condition at infinity.

In order to precisely state our results we denote by $\mathcal{F}$ the class of functions $h \in C\left(\mathbb{R}^{N}, \mathbb{R}\right) \cap L^{\infty}\left(\mathbb{R}^{N}, \mathbb{R}\right)$ such that, for every $\varepsilon>0$, the set $\left\{x \in \mathbb{R}^{N}:|h(x)| \geq \varepsilon\right\}$ has finite Lebesgue measure. We suppose that $V$ is a perturbation of a periodic function at the infinity in the following sense:

$(V)$ there exist a constant $a_{0}>0$ and a function $V_{0} \in C\left(\mathbb{R}^{N}, \mathbb{R}\right)$, 1-periodic in $x_{i}, 1 \leq i \leq N$, such that $V_{0}-V \in \mathcal{F}$ and

$$
V_{0}(x) \geq V(x) \geq a_{0}>0, \text { for all } x \in \mathbb{R}^{N} .
$$

Considering $F(x, t)=\int_{0}^{t} f(x, s) d s$ the primitive of $f \in C\left(\mathbb{R}^{N} \times \mathbb{R}, \mathbb{R}\right)$, we also suppose the following hypotheses:

$\left(f_{1}\right) F(x, t) \geq 0$ for all $(x, t) \in \mathbb{R}^{N} \times \mathbb{R}$ and $f(x, t)=o(t)$ uniformly in $x \in \mathbb{R}^{N}$ as $t \rightarrow 0$;

$\left(f_{2}\right)$ there exists a function $b \in C\left(\mathbb{R} \backslash\{0\}, \mathbb{R}^{+}\right)$such that

$$
\widehat{F}(x, t):=\frac{1}{2} f(x, t) t-F(x, t) \geq b(t) t^{2},
$$

for all $(x, t) \in \mathbb{R}^{N} \times \mathbb{R}$;

$\left(f_{3}\right)$ there exist $a_{1}>0, R_{1}>0$ and $\tau>\max \{1, N / 2\}$ such that

$$
|f(x, t)|^{\tau} \leq a_{1}|t|^{\tau} \widehat{F}(x, t),
$$

for all $(x, t)$ with $|t|>R_{1}$; 
$\left(f_{4}\right)$ uniformly in $x \in \mathbb{R}^{N}$ it holds

$$
\lim _{|t| \rightarrow+\infty} \frac{F(x, t)}{t^{2}}=+\infty
$$

$\left(f_{5}\right)$ there exist $q \in\left(2,2^{*}\right)$ and functions $h \in \mathcal{F}, f_{0} \in C\left(\mathbb{R}^{N} \times \mathbb{R}, \mathbb{R}\right)$, 1-periodic in $x_{i}, 1 \leq i \leq N$, such that:

(i) $F(x, t) \geq F_{0}(x, t)=\int_{0}^{t} f_{0}(x, s) d s$, for all $(x, t) \in \mathbb{R}^{N} \times \mathbb{R}$;

(ii) $\left|f(x, t)-f_{0}(x, t)\right| \leq h(x)|t|^{q-1}$, for all $(x, t) \in \mathbb{R}^{N} \times \mathbb{R}$;

(iii) $\frac{f_{0}(x, \cdot)}{|\cdot|}$ is increasing in $\mathbb{R} \backslash\{0\}$, for all $x \in \mathbb{R}^{N}$.

The main result of this paper can be stated as follows:

Theorem 1.1. Suppose that $V$ and $f$ satisfy $(V)$ and $\left(f_{1}\right)-\left(f_{5}\right)$, respectively. Then the problem (1.1) possesses a solution.

As a by product of our calculations we can obtain a weak solution for the periodic problem. In this setting we can drop the condition $\left(f_{5}\right)$, and we shall prove the following result:

Theorem 1.2. Suppose that $V(\cdot)$ and $f(\cdot, t)$ are 1-periodic in $x_{i}, 1 \leq i \leq N$, and $V(x) \geq a_{0}>0$ for all $x \in \mathbb{R}^{N}$. If $f$ satisfies $\left(f_{1}\right),\left(f_{3}\right),\left(f_{4}\right)$ and

$\left(f_{2}\right)^{\prime} \widehat{F}(x, t)>0$ for all $t \neq 0$,

then the problem (1.1) possesses a ground states solution.

Problems as (1.1) has been focus of intensive research in recent years. Initially, several authors have dealt with the case where $f$ behaves like $q(x)|u|^{p-1} u, 1<$ $p<2^{*}-1$ and $V$ is constant (see [5, 4). In the work of Rabinowitz 11 and Rabinowitz-Coti Zelati [15] it was imposed the classical superlinear condition due to Ambrosetti-Rabinowitz:

$(A R)$ there exists $\mu>2$ such that

$$
0<\mu F(x, t) \leq f(x, t) t
$$

for all $x \in \mathbb{R}^{N}$ and $t \neq 0$.

This hypothesis has a important role to show that (PS) sequences are bounded. In this work we assume the condition $\left(f_{4}\right)$ which is weaker than the condition of $(A R)$. It has already appeared in the papers of Ding-Lee [7] and Ding-Szulkin [8].

We emphasize that, in Theorem 1.1. we are not supposing periodicity on $V$ or $f(\cdot, t)$. Instead, we consider the asymptotically periodic case as done in the paper of Lins-Silva 9. The condition $\left(f_{5}\right)$ describes our assumption of asymptotically periodic for the nonlinearity $f$. A pioneering work on problems as (1.1) is due Alama-Li [1] that focused the case $V \equiv 1$ and $f$ asymptotically periodic in a weaker sense. We also cite the papers [2, 3, 9, 13, 12, for some related (and not comparable) results.

As an example of application of our main theorem we take $a \in C\left(\mathbb{R}^{N}, \mathbb{R}\right) \cap$ $L^{\infty}\left(\mathbb{R}^{N}, \mathbb{R}\right) 1$-periodic in $x_{i}, 1 \leq i \leq N$ with $a(x) \geq 2$. Define the functions

$$
\begin{gathered}
f(x, t)=a(x) t \ln (1+t)+e^{-|x|^{2}} t(\ln (1+t)+1-\cos (t)), t \geq 0, \\
f_{0}(x, t)=a(x) t \ln (1+t), t \geq 0,
\end{gathered}
$$

and $f(x, t)=-f(x,-t), f_{0}(x, t)=-f_{0}(x,-t)$ for $t<0$. This function satisfies $\left(f_{1}\right)-\left(f_{5}\right)$, but not satisfies $(A R)$. Moreover $f(x, t) / t$ is oscillatory, and therefore the Nehari approach used in [14] is not applicable. 
The rest of the article is organized as follows. In Section 2 we present the technical results that be used throughout the work. The final Section 3 is devoted to the proof of Theorems 1.1 and 1.2

\section{Preliminary Results}

In this section we present some preliminaries for the proofs of our main theorems. We denote by $B_{R}(y)$ the open ball in $\mathbb{R}^{N}$ of radius $R>0$ and center at the point $y$. The Lebesgue measure of a set $A \subset \mathbb{R}^{N}$ will be denoted by $|A|$. To shorten notation, write $\int_{A} u$ instead of $\int_{A} u(x) d x$. We also omit the set $A$ whenever $A=\mathbb{R}^{N}$. We write $|\cdot|_{p}$ for the norm in $L^{p}\left(\mathbb{R}^{N}\right)$.

Throughout the paper we assume that the potential $V$ satisfies the assumption $(V)$. This implies that the norm

$$
\|u\|^{2}=\int\left(|\nabla u|^{2}+V(x) u^{2}\right), u \in H^{1}\left(\mathbb{R}^{N}\right)
$$

is equivalent to the usual one. In what follows we denote by $H$ the space $H^{1}\left(\mathbb{R}^{N}\right)$ endowed with the above norm.

In our first lemma we obtain the basic estimates on the behavior of the nonlinearity $f$.

Lemma 2.1. Suppose that $f$ satisfies $\left(f_{1}\right),\left(f_{3}\right)$ and $\left(f_{5}\right)(i i)$. Then, for any given $\varepsilon>0$, there exists $C_{\varepsilon}>0$ and $p \in\left(2,2^{*}\right)$ such that

$$
|f(x, t)| \leq \varepsilon|t|+C_{\varepsilon}|t|^{p-1}, \quad|F(x, t)| \leq \varepsilon|t|^{2}+C_{\varepsilon}|t|^{p},
$$

for all $(x, t) \in \mathbb{R}^{N} \times \mathbb{R}$.

Proof. Taking $\varepsilon>0$ and using $\left(f_{1}\right)$, we obtain $\delta>0$ such that

$$
|f(x, t)| \leq \varepsilon|t|, x \in \mathbb{R}^{N},|t| \leq \delta .
$$

By $\left(f_{3}\right)$ there exists $R>0$ satisfying

$$
|f(x, t)|^{\tau} \leq a_{1}|t|^{\tau} \widehat{F}(x, t) \leq \frac{a_{1}}{2}|t|^{\tau+1}|f(x, t)|, x \in \mathbb{R}^{N},|t| \geq R .
$$

Then, setting $p=2 \tau /(\tau-1)$, we can use $\tau>N / 2$ to conclude that $2<p<2^{*}$. Moreover,

$$
|f(x, t)| \leq C|t|^{\frac{\tau+1}{\tau-1}}=C|t|^{p-1}, x \in \mathbb{R}^{N},|t| \geq R
$$

From the continuity and periodicity of $f_{0}$ we obtain $M>0$ such that

$$
\left|f_{0}(x, t)\right| \leq M, x \in \mathbb{R}^{N}, \delta \leq|t| \leq R
$$

Now, using $\left(f_{5}\right)(i i)$ we get

$$
|f(x, t)| \leq\|h\|_{\infty}|t|^{q-1}+M \leq\left(\|h\|_{\infty}+\frac{M}{\delta^{q-1}}\right)|t|^{q-1}, x \in \mathbb{R}^{N}, \delta \leq|t| \leq R .
$$

This, (2.2) and (2.3) proves the first inequality in (2.1). The second one follows directly by integration.

In view of the above lemma it is well defined the functional $I: H \rightarrow \mathbb{R}$ given by

$$
I(u)=\frac{1}{2}\|u\|^{2}-\int F(x, u) .
$$


Moreover, standard calculations show that $I \in C^{1}(H, \mathbb{R})$ and the Gateaux derivative of $I$ has the following form

$$
I^{\prime}(u) v=\int(\nabla u \nabla v+V(x) u v)-\int f(x, u) v,
$$

for any $u, v \in H$. Hence, the critical points de $I$ are precisely the weak solutions of the problem (1.1).

In order to obtain the desired critical points we shall use the following abstract result. We refer to [9, Theorem 2.3].

Theorem 2.2 (Local Mountain Pass Theorem). Let $E$ be a real Banach space. Suppose that $I \in C^{1}(E, \mathbb{R})$ satisfies $I(0)=0$ and

$\left(I_{1}\right)$ there exist $\rho, \alpha>0$ such that $I(u) \geq \alpha>0$ for all $\|u\|=\rho$,

$\left(I_{2}\right)$ there exist $e \in E$ with $\|e\|>\rho$ such that $I(e) \leq 0$.

If there exists $\gamma_{0} \in \Gamma=\{\gamma \in C([0,1], E): \gamma(0)=0,\|\gamma(1)\|>\rho, I(\gamma(1)) \leq 0\}$ such that

$$
c=\max _{t \in[0,1]} I\left(\gamma_{0}(t)\right)>0
$$

then I possesses a nontrivial critical point $u \in \gamma_{0}([0,1])$ at the level $c$.

In the next result we prove that the functional $I$ verifies the geometric conditions of the Mountain Pass Theorem.

Lemma 2.3. Suppose that $f$ satisfies $\left(f_{1}\right),\left(f_{3}\right),\left(f_{4}\right)$ and $\left(f_{5}\right)(i i)$. Then I satisfies $\left(I_{1}\right)$ and $\left(I_{2}\right)$.

Proof. By Lemma 2.1 and Sobolev inequality we have

$$
\int F(x, u) \leq \varepsilon|u|_{2}^{2}+C_{\varepsilon}|u|_{p}^{p} \leq c_{1} \varepsilon\|u\|^{2}+C\|u\|^{p},
$$

for some $c_{1}>0$. Since $p>2$, we have

$$
I(u) \geq\left(\frac{1}{2}-c_{1} \varepsilon\right)\|u\|^{2}+o\left(\|u\|^{2}\right) \geq \alpha
$$

for $\|u\|=\rho$ small enough. This proves $\left(I_{1}\right)$.

In order to verify the condition $\left(I_{2}\right)$ we fix $\varphi \in C_{0}^{\infty}\left(\mathbb{R}^{N}\right)$ satisfying $\varphi(x) \geq 0$ in $\mathbb{R}^{N}$ and $\|\varphi\|=1$. We claim that there is $R_{0}>0$ such that, for any $R>R_{0}$, we have that $I(R \varphi)<0$. If this is true it suffices to take $e=R \varphi$ with $R>0$ large enough to get $\left(I_{2}\right)$.

For the proof of the claim we set $k=2 / \int \varphi^{2}$ and use $\left(f_{4}\right)$ to obtain $M>0$ satisfying

$$
F(x, t) \geq k t^{2} \text { for all }|t| \geq M .
$$

Hence, setting $A_{R}=\left\{x \in \mathbb{R}^{N} ; \varphi(x) \geq M / R\right\}$, we get

$$
\int F(x, R \varphi) \geq \int_{A_{R}} F(x, R \varphi) \geq k R^{2} \int_{A_{R}} \varphi^{2} .
$$

Since $\varphi \geq 0$ we can choose $R_{0}>0$ such that, for any $R \geq R_{0}$, it holds $\int_{A_{R}} \varphi^{2} \geq$ $\frac{1}{2} \int \varphi^{2}$. It follows from the definition of $k$ and (2.4) that $\int F(x, R \varphi) \geq R^{2}$ and therefore

for any $R>R_{0}$.

$$
I(R \varphi) \leq \frac{1}{2} R^{2}-R^{2}=-\frac{1}{2} R^{2}<0,
$$


We recall that $I$ is said to satisfy the Cerami condition at the level $c \in \mathbb{R}$ if any sequence $\left(u_{n}\right) \subseteq H$ such that

$$
\lim _{n \rightarrow+\infty} I\left(u_{n}\right)=c \text { and } \lim _{n \rightarrow+\infty}\left(1+\left\|u_{n}\right\|_{E}\right)\left\|I^{\prime}\left(u_{n}\right)\right\|_{H^{\prime}}=0
$$

possesses a convergent subsequence in $H$. A sequence $\left(u_{n}\right) \subseteq H$ as above is called Cerami sequence for $I$.

Lemma 2.4. Suppose that $f$ satisfies $\left(f_{1}\right)-\left(f_{4}\right)$ and $\left(f_{5}\right)(i i)$. Then any Cerami sequence for $I$ is bounded.

Proof. We adapt here an argument from [7]. Let $\left(u_{n}\right) \subset H$ be such that

$$
\lim _{n \rightarrow+\infty} I\left(u_{n}\right)=c \text { and } \lim _{n \rightarrow+\infty}\left(1+\left\|u_{n}\right\|\right)\left\|I^{\prime}\left(u_{n}\right)\right\|_{H^{\prime}}=0 .
$$

It follows that

$$
c+o_{n}(1)=I\left(u_{n}\right)-\frac{1}{2} I^{\prime}\left(u_{n}\right) u_{n}=\int \widehat{F}\left(x, u_{n}\right),
$$

where $o_{n}(1)$ stands for a quantity approaching zero as $n \rightarrow+\infty$. Suppose by contradiction that, for some subsequence still denote $\left(u_{n}\right)$, we have that $\left\|u_{n}\right\| \rightarrow \infty$. By defining $v_{n}=\frac{u_{n}}{\left\|u_{n}\right\|}$ we obtain

$$
o_{n}(1)=\frac{I^{\prime}\left(u_{n}\right) u_{n}}{\left\|u_{n}\right\|^{2}}=1-\int \frac{f\left(x, u_{n}\right) v_{n}}{\left\|u_{n}\right\|}
$$

and therefore

$$
\lim _{n \rightarrow+\infty} \int \frac{f\left(x, u_{n}\right) v_{n}}{\left\|u_{n}\right\|}=1 .
$$

For any $r \geq 0$ we set

$$
g(r)=\inf \left\{\widehat{F}(x, t) ; x \in \mathbb{R}^{N},|t| \geq r\right\} .
$$

Let $R_{1}>0$ be given from $\left(f_{3}\right)$. For any $|t|>R_{1}$, there holds

$$
a_{1} \widehat{F}(x, t) \geq\left(\frac{f(x, t)}{t}\right)^{\tau} \geq\left(\frac{2 F(x, t)}{t^{2}}\right)^{\tau} .
$$

Hence, it follows from $\left(f_{4}\right)$ that $\widehat{F}(x, t) \rightarrow \infty$ as $t \rightarrow \infty$ uniformly in $x \in \mathbb{R}^{N}$. This, $\left(f_{2}\right)$ and the definition of $g$ imply that $g(r)>0$ for all $r>0$ and $g(r) \rightarrow \infty$ as $r \rightarrow \infty$.

For $0 \leq a<b$, we define

$$
\Omega_{n}(a, b)=\left\{x \in \mathbb{R}^{N} ; a \leq\left|u_{n}(x)\right|<b\right\}
$$

and for $a>0$,

$$
c_{a}^{b}=\inf \left\{\frac{\widehat{F}(x, t)}{t^{2}} ; x \in \mathbb{R}^{N}, a \leq|t| \leq b\right\} .
$$

From $\left(f_{2}\right)$ we have that $c_{a}^{b}>0$. By using (2.5) and the above definitions we obtain

$$
\begin{aligned}
c+o_{n}(1) & =\int_{\Omega_{n}(0, a)} \widehat{F}\left(x, u_{n}\right)+\int_{\Omega_{n}(a, b)} \widehat{F}\left(x, u_{n}\right)+\int_{\Omega_{n}(b, \infty)} \widehat{F}\left(x, u_{n}\right) \\
& \geq \int_{\Omega_{n}(0, a)} \widehat{F}\left(x, u_{n}\right)+c_{a}^{b} \int_{\Omega_{n}(a, b)} u_{n}^{2}+g(b)\left|\Omega_{n}(b, \infty)\right|,
\end{aligned}
$$


and therefore, for some $C_{1}>0$, we have that

$$
\max \left\{\int_{\Omega_{n}(0, a)} \widehat{F}\left(x, u_{n}\right), c_{a}^{b} \int_{\Omega_{n}(a, b)} u_{n}^{2}, g(b)\left|\Omega_{n}(b, \infty)\right|\right\} \leq C_{1}
$$

The above inequality implies that $\left|\Omega_{n}(b, \infty)\right| \leq C / g(b)$. Recalling that $g(b) \rightarrow$ $+\infty$ as $b \rightarrow+\infty$ we conclude that

$$
\lim _{b \rightarrow+\infty}\left|\Omega_{n}(b, \infty)\right|=0
$$

Fixed $\mu \in\left[2,2^{*}\right)$ and $\nu \in\left(\mu, 2^{*}\right)$, by Hölder's inequality and Sobolev embedding, we obtain, for some $C_{2}>0$,

$$
\begin{aligned}
\int_{\Omega_{n}(b, \infty)}\left|v_{n}\right|^{\mu} & \leq\left(\int_{\Omega_{n}(b, \infty)}\left|v_{n}\right|^{\nu}\right)^{\mu / \nu}\left|\Omega_{n}(b, \infty)\right|^{(\nu-\mu) / \nu} \\
& \leq C_{2}\left\|v_{n}\right\|^{\mu}\left|\Omega_{n}(b, \infty)\right|^{(\nu-\mu) / \nu}=C_{2}\left|\Omega_{n}(b, \infty)\right|^{(\nu-\mu) / \nu} .
\end{aligned}
$$

Since $\nu-\mu>0$ we conclude that

$$
\lim _{b \rightarrow+\infty} \int_{\Omega_{n}(b, \infty)}\left|v_{n}\right|^{\mu}=0 .
$$

Again from (2.7), for $0<a<b$ fixed, it follows that

$$
\int_{\Omega_{n}(a, b)}\left|v_{n}\right|^{2}=\frac{1}{\left\|u_{n}\right\|^{2}} \int_{\Omega_{n}(a, b)} u_{n}^{2} \leq \frac{1}{\left\|u_{n}\right\|^{2}} \frac{C_{1}}{c_{a}^{b}}=o_{n}(1) .
$$

Let $C_{3}>0$ be such that $|u|_{2} \leq C_{3}\|u\|$ for all $u \in H$ and consider $\varepsilon \in(0,1 / 3)$. By $\left(f_{1}\right)$, there exists $a_{\varepsilon}>0$ such that

$$
|f(x, u)| \leq \frac{\varepsilon|u|}{C_{3}^{2}} \text { for all }|u| \leq a_{\varepsilon} .
$$

Hence,

$$
\int_{\Omega_{n}\left(0, a_{\varepsilon}\right)} \frac{f\left(x, u_{n}\right) v_{n}}{\left\|u_{n}\right\|} \leq \frac{\varepsilon}{C_{3}^{2}} \int_{\Omega_{n}\left(0, a_{\varepsilon}\right)} v_{n}^{2} \leq \varepsilon
$$

Using $\left(f_{5}\right)$ and recalling that $h \in L^{\infty}\left(\mathbb{R}^{N}, \mathbb{R}\right)$ we obtain $C_{4}>0$ such that $\left|f\left(x, u_{n}\right)\right| \leq$ $C_{4}\left|u_{n}\right|$ for every $x \in \Omega_{n}\left(a_{\varepsilon}, b_{\varepsilon}\right)$ and so,

$$
\int_{\Omega_{n}\left(a_{\varepsilon}, b_{\varepsilon}\right)} \frac{f\left(x, u_{n}\right) v_{n}}{\left\|u_{n}\right\|} \leq C_{4} \int_{\Omega_{n}\left(a_{\varepsilon}, b_{\varepsilon}\right)} v_{n}^{2}<\varepsilon, \text { for all } n \geq n_{0} .
$$


If we set $2 \tau^{\prime}=2 \tau /(\tau-1) \in\left(2,2^{*}\right)$, we can use condition $\left(f_{3}\right)$, (2.7) and Hölder's inequality to get

$$
\begin{aligned}
\int_{\Omega_{n}\left(b_{\varepsilon}, \infty\right)} \frac{f\left(x, u_{n}\right) v_{n}}{\left\|u_{n}\right\|} & =\int_{\Omega_{n}\left(b_{\varepsilon}, \infty\right)} \frac{f\left(x, u_{n}\right) v_{n}^{2}}{\left|u_{n}\right|} \\
& \leq\left(\int_{\Omega_{n}\left(b_{\varepsilon}, \infty\right)} \frac{\left|f\left(x, u_{n}\right)\right|^{\tau}}{\left|u_{n}\right|^{\tau}}\right)^{1 / \tau}\left(\int_{\Omega_{n}\left(b_{\varepsilon}, \infty\right)}\left|v_{n}\right|^{2 \tau^{\prime}}\right)^{1 / \tau^{\prime}} \\
& \leq a_{1}^{1 / \tau}\left(\int_{\Omega_{n}\left(b_{\varepsilon}, \infty\right)} \widehat{F}\left(x, u_{n}\right)\right)^{1 / \tau}\left(\int_{\Omega_{n}\left(b_{\varepsilon}, \infty\right)}\left|v_{n}\right|^{2 \tau^{\prime}}\right)^{1 / \tau^{\prime}} \\
& \leq C_{1}\left(\int_{\Omega_{n}\left(b_{\varepsilon}, \infty\right)}\left|v_{n}\right|^{2 \tau^{\prime}}\right)^{1 / \tau^{\prime}}
\end{aligned}
$$

This expression and (2.9) provides $b_{\varepsilon}>0$ large in such way that

$$
\int_{\Omega_{n}\left(b_{\varepsilon}, \infty\right)} \frac{f\left(x, u_{n}\right) v_{n}}{\left\|u_{n}\right\|}<\varepsilon, \text { for all } n \geq n_{0} .
$$

Finally, the estimates (2.10) - (2.12) imply

$$
\int \frac{f\left(x, u_{n}\right) v_{n}}{\left\|u_{n}\right\|} \leq 3 \varepsilon<1
$$

which contradicts (2.6). Therefore $\left(u_{n}\right)$ is bounded in $H$.

Remark 2.5. If $f$ is periodic we can obtain the estimate in (2.11) without the condition $\left(f_{5}\right)$. Moreover, in this case, it follows from periodicity and continuity of $F_{0}$ that $\frac{F_{0}(x, u)}{u^{2}} \geq k=k(a, b)>0$ for all $x \in \Omega_{n}(a, b)$. Of course $c_{a}^{b} \geq k>0$ and therefore the above lemma holds under the setting of Theorem 1.2.

Lemma 2.6. Suppose that $f$ satisfies $\left(f_{1}\right)$ and $\left(f_{2}\right)$. Let $\left(u_{n}\right) \subset H$ be a Cerami sequence for $I$ at level $c>0$. If $u_{n} \rightarrow 0$ weakly in $H$ then there exist a sequence $\left(y_{n}\right) \subset \mathbb{R}^{N}$ and $R>0, \alpha>0$ such that $\left|y_{n}\right| \rightarrow \infty$ and

$$
\limsup _{n \rightarrow \infty} \int_{B_{R}\left(y_{n}\right)}\left|u_{n}\right|^{2} \geq \alpha>0
$$

Proof. Suppose, by contradiction, that the lemma is false. Then, for any $R>0$, we have that

$$
\limsup _{n \rightarrow \infty} \int_{B_{R}(y)}\left|u_{n}\right|^{2}=0 \text { for all } R>0 .
$$

Hence, we can use a result of Lions (see [10]) to conclude that $\left|u_{n}\right|_{s} \rightarrow 0$ for any $s \in\left(2,2^{*}\right)$. It follows from the second inequality in (2.1) that

$$
\limsup _{n \rightarrow+\infty} \int F\left(x, u_{n}\right) \leq \limsup _{n \rightarrow \infty}\left(\varepsilon \int\left|u_{n}\right|^{2}+C_{\varepsilon} \int\left|u_{n}\right|^{p}\right) \leq C \varepsilon
$$

where we have used the boundedness of $\left(u_{n}\right)$ in $L^{2}\left(\mathbb{R}^{N}\right)$. Since $\varepsilon$ is arbitrary we conclude that $\int F\left(x, u_{n}\right) \rightarrow 0$ as $n \rightarrow+\infty$. The same argument and the first inequality in (2.1) imply that $\int f\left(x, u_{n}\right) u_{n} \rightarrow 0$ as $n \rightarrow+\infty$. 
Since $\left(u_{n}\right)$ is a Cerami sequence, we get

$$
c=\lim _{n \rightarrow \infty}\left[I\left(u_{n}\right)-\frac{1}{2} I^{\prime}\left(u_{n}\right) u_{n}\right]=\lim _{n \rightarrow \infty} \int\left(\frac{1}{2} f\left(x, u_{n}\right) u_{n}-F\left(x, u_{n}\right)\right)=0
$$

which contradicts $c>0$. The lemma is proved.

We finish the section by stating two technical convergence results. The proofs can be found in [9, Lemmas 5.1 and 5.2], respectively.

Lemma 2.7. Suppose that $(V)$ and $\left(f_{5}\right)$ are satisfied. Let $\left(u_{n}\right) \subset H$ be a bounded sequence and $v_{n}(x)=v\left(x-y_{n}\right)$, where $v \in H$ and $\left(y_{n}\right) \subset \mathbb{R}^{N}$. If $\left|y_{n}\right| \rightarrow \infty$, then we have

$$
\begin{gathered}
{\left[V_{0}(x)-V(x)\right] u_{n} v_{n} \rightarrow 0,} \\
{\left[f_{0}\left(x, u_{n}\right)-f\left(x, u_{n}\right)\right] v_{n} \rightarrow 0,}
\end{gathered}
$$

strongly in $L^{1}\left(\mathbb{R}^{N}\right)$, as $n \rightarrow \infty$.

Lemma 2.8. Suppose $h \in \mathcal{F}$ and $s \in\left[2,2^{*}\right]$. If $\left(v_{n}\right) \subseteq H^{1}\left(\mathbb{R}^{N}\right)$ is such that $v_{n} \rightarrow v$ weakly in $H$, then

$$
\lim _{n \rightarrow+\infty} \int h\left|v_{n}\right|^{s}=\int h|v|^{s} .
$$

\section{Proofs of the main Results}

In section, we denote by $I_{0}: H \rightarrow \mathbb{R}$ the functional associated with the periodic problem, namely

$$
I_{0}(u)=\frac{1}{2} \int\left(|\nabla u|^{2}+V_{0}(x) u^{2}\right)-\int F_{0}(x, u) .
$$

We also consider the following norm in $H^{1}\left(\mathbb{R}^{N}\right)$

$$
\|u\|_{0}=\left(\int\left(|\nabla u|^{2}+V_{0}(x) u^{2}\right)^{2},\right.
$$

which is equivalent to the usual norm of this space.

We are ready to prove our main theorem as follows:

Proof of Theorem 1.1. By Lemma 2.3 and the Mountain Pass Theorem there exists a sequence $\left(u_{n}\right) \subset H$ such that

$$
I\left(u_{n}\right) \rightarrow c \geq \alpha>0 \text { and }\left(1+\left\|u_{n}\right\|\right) I^{\prime}\left(u_{n}\right) \rightarrow 0, \text { as } n \rightarrow \infty .
$$

Applying Lemma 2.4 we may assume, without loss generality, that $u_{n} \rightarrow u$ weakly in $H$. We claim that $I^{\prime}(u)=0$. Indeed, since $C_{0}^{\infty}\left(\mathbb{R}^{N}\right)$ is dense in $H$, it suffices to show that $I^{\prime}(u) \varphi=0$ for all $\varphi \in C_{0}^{\infty}\left(\mathbb{R}^{N}\right)$. We have

$$
I^{\prime}\left(u_{n}\right) \varphi-I^{\prime}(u) \varphi=o_{n}(1)-\int\left[f\left(x, u_{n}\right)-f(x, u)\right] \varphi .
$$

Using the Sobolev embedding theorem we can assume that, up to a subsequence, $u_{n} \rightarrow u$ in $L_{l o c}^{s}\left(\mathbb{R}^{N}\right)$ for each $s \in\left[1,2^{*}\right)$ and

$$
\begin{aligned}
& u_{n}(x) \rightarrow u(x) \text { a.e. on } K, \text { as } n \rightarrow \infty, \\
& \left|u_{n}(x)\right| \leq w_{s}(x) \in L^{s}(K), \text { for every } n \in \mathbb{N} \text { and a.e. on } K,
\end{aligned}
$$

where $K$ denotes the support of the function $\varphi$. Therefore,

$$
f\left(x, u_{n}\right) \rightarrow f(x, u) \text { a.e. on } K, \text { as } n \rightarrow \infty,
$$


and using (2.1), we get

$$
\left|f\left(x, u_{n}\right) \varphi\right| \leq \varepsilon\left|w_{2}\right||\varphi|+C_{\varepsilon}\left|w_{p-1}\right||\varphi| \in L^{1}(K) .
$$

Thus, taking the limit in (3.2) and using the Lebesgue Dominated Convergence we get

$$
I^{\prime}(u) \varphi=\lim _{n \rightarrow \infty} I^{\prime}\left(u_{n}\right) \varphi=0
$$

which implies $I^{\prime}(u)=0$.

If $u \neq 0$, the theorem is proved. So, we deal in the sequel with the case $u=0$. By Lemma 2.6. we recall that there exist a sequence $\left(y_{n}\right) \subset \mathbb{R}^{N}, R>0$, and $\alpha>0$ such that $\left|y_{n}\right| \rightarrow \infty$ as $n \rightarrow \infty$, and

$$
\limsup _{n \rightarrow \infty} \int_{B_{R}\left(y_{n}\right)}\left|u_{n}\right|^{2} \geq \alpha>0
$$

Without loss of generality we may assume that $\left(y_{n}\right) \subset \mathbb{Z}^{N}$ (see [6, page 7]). Writing $\widetilde{u}_{n}(x)=u_{n}\left(x+y_{n}\right)$ and observing that $\left\|\widetilde{u}_{n}\right\|=\left\|u_{n}\right\|_{0}$, up to subsequence we have $\widetilde{u}_{n} \rightarrow \widetilde{u}$ in $H, \widetilde{u}_{n} \rightarrow \widetilde{u}$ in $L_{l o c}^{2}\left(\mathbb{R}^{N}\right)$ and for almost every $x \in \mathbb{R}^{N}$. From (3.3), we have $\widetilde{u} \neq 0$.

Claim 1. $I_{0}^{\prime}(\widetilde{u})=0$

To prove the claim we take $\varphi \in C_{0}^{\infty}\left(\mathbb{R}^{N}\right)$ and define, for each $n \in \mathbb{N}, \varphi_{n}(x)=$ $\varphi\left(x-y_{n}\right)$. Arguing as in the beginning of the proof and using the periodicity of $f_{0}$ we get

$$
I_{0}^{\prime}(\widetilde{u}) \varphi=I_{0}^{\prime}\left(\widetilde{u}_{n}\right) \varphi+o_{n}(1)=I_{0}^{\prime}\left(u_{n}\right) \varphi_{n}+o_{n}(1),
$$

and therefore it suffices to check that $I_{0}^{\prime}\left(u_{n}\right) \varphi_{n}=o_{n}(1)$. To achieve this objective we notice that, by Lemma 2.7 .

$$
\begin{aligned}
I_{0}^{\prime}\left(u_{n}\right) \varphi_{n} & =I^{\prime}\left(u_{n}\right) \varphi_{n}+\int\left[V_{0}(x)-V(x)\right] u_{n} \varphi_{n}-\int\left[f_{0}\left(x, u_{n}\right)-f(x, u)\right] \varphi_{n} \\
& =I^{\prime}\left(u_{n}\right) \varphi_{n}+o_{n}(1) .
\end{aligned}
$$

So, by (3.1), the claim is verified.

Claim 2. $\liminf _{n \rightarrow \infty} \int \widehat{F}\left(x, u_{n}\right) \geq \int \widehat{F}_{0}(x, \widetilde{u})$

By using $\left(f_{5}\right)(i i)$ and a straightforward calculation we obtain

$$
\left|\widehat{F}(x, t)-\widehat{F}_{0}(x, t)\right| \leq\left(\frac{1}{2}+\frac{1}{q}\right) h(x)|t|^{q} .
$$

Since $u_{n} \rightarrow 0$ weakly in $H$, it follows from the above inequality and Lemma 2.8 that

$$
\begin{aligned}
\lim _{n \rightarrow \infty} \int \widehat{F}\left(x, u_{n}\right) & =\lim _{n \rightarrow \infty} \int \widehat{F}_{0}\left(x, u_{n}\right) \\
& =\liminf _{n \rightarrow \infty} \int \widehat{F}_{0}\left(x, \widetilde{u}_{n}\right) \geq \int \widehat{F}_{0}(x, \widetilde{u}),
\end{aligned}
$$

where we also have used the periodicity of $\widehat{F}_{0}$. 
By using (3.1) and the above claim we get

$$
\begin{aligned}
c & =\lim _{n \rightarrow \infty}\left[I\left(u_{n}\right)-\frac{1}{2} I^{\prime}\left(u_{n}\right) u_{n}\right]=\liminf _{n \rightarrow \infty} \int \widehat{F}\left(x, u_{n}\right) \\
& \geq \int \widehat{F}_{0}(x, \widetilde{u})=I_{0}(\widetilde{u})-\frac{1}{2} I_{0}^{\prime}(\widetilde{u}) \widetilde{u}=I_{0}(\widetilde{u}),
\end{aligned}
$$

and therefore $I_{0}(\widetilde{u}) \leq c$. It follows from $\left(f_{5}\right)(i i i)$ that $\max _{t \geq 0} I_{0}(t \widetilde{u})=I_{0}(\widetilde{u})$. Hence, by the definition of $c,(V)$ and $\left(f_{5}\right)(i)$, we have that

$$
c \leq \max _{t \geq 0} I(t \widetilde{u}) \leq \max _{t \geq 0} I_{0}(t \widetilde{u})=I_{0}(\widetilde{u}) \leq c
$$

We can now invoke Theorem 2.2 to conclude that $I$ possesses a critical point at level $c>0$. This finishes the proof.

We proceed now with the proof of the periodic result.

Proof of Theorem 1.2. We first notice that Lemmas 2.1, 2.2 and 2.3 are still valid under the assumptions of the Theorem 1.2 Hence, by Lemma 2.3 . we obtain a sequence $\left(u_{n}\right) \subset H$ such that

$$
\lim _{n \rightarrow+\infty} I_{0}\left(u_{n}\right)=c_{0} \quad \text { and } \lim _{n \rightarrow+\infty}\left(1+\left\|u_{n}\right\|_{0}\right)\left\|I_{0}^{\prime}\left(u_{n}\right)\right\|=0,
$$

where $c_{0}$ is the mountain-pass level of $I_{0}$. Arguing as in the proof of Theorem 1.1 we conclude that $u_{n} \rightarrow u$ weakly in $H$ with $I_{0}^{\prime}(u)=0$.

As before, we need only consider the case $u=0$. By the Lemma 2.6] there is a sequence $\left(y_{n}\right) \subset \mathbb{Z}^{N}$ (see [6, page 7]), $R>0$ and $\alpha>0$ such that $\left|y_{n}\right| \rightarrow \infty$ as $n \rightarrow \infty$ and

$$
\limsup _{n \rightarrow \infty} \int_{B_{R}\left(y_{n}\right)}\left|u_{n}\right|^{2} \geq \alpha>0
$$

Writing $\widetilde{u}_{n}(x)=u_{n}\left(x+y_{n}\right)$ and observing that $\left\|\widetilde{u}_{n}\right\|_{0}=\left\|u_{n}\right\|_{0}$, up to subsequence, we have $\widetilde{u}_{n} \rightarrow \widetilde{u}$ weakly in $H, \widetilde{u}_{n} \rightarrow \widetilde{u}$ in $L_{l o c}^{2}\left(\mathbb{R}^{N}\right)$ and $\widetilde{u}_{n}(x) \rightarrow \widetilde{u}(x)$ almost everywhere in $\mathbb{R}^{N}$. The local convergence and (3.4) imply that $\widetilde{u} \neq 0$. Arguing as in Claim 1 of the proof of Theorem 1.1 we conclude that $I_{0}^{\prime}(\widetilde{u})=0$ and therefore we obtain a nonzero weak solution.

In view of the above existence result it is well defined

$$
m=\inf \left\{I_{0}(u) ; u \in E \text { and } I^{\prime}(u)=0\right\}>0 .
$$

We claim that $m$ is achieved. Indeed, let $\left(u_{n}\right) \subset H$ be a minimizing sequence for $m$, namely

$$
I_{0}\left(u_{n}\right) \rightarrow m, I_{0}^{\prime}\left(u_{n}\right)=0 \text { and } u_{n} \neq 0 .
$$

Since $\left(u_{n}\right)$ is a Cerami sequence for $I_{0}$ it follows from Lemma 2.4 that it is bounded. Moreover, using $I_{0}^{\prime}\left(u_{n}\right) u_{n}=0$ and (2.1) with $\varepsilon$ small, we can obtain $k>0$ satisfying $\left\|u_{n}\right\|_{0} \geq k$. Thus, arguing as in the preceding paragraph, we obtain a translated subsequence $\left(\widetilde{u}_{n}\right)$ which has a nonzero weak limit $u_{0}$ such that $I_{0}^{\prime}\left(u_{0}\right)=0$ and $\widetilde{u}_{n}(x) \rightarrow u_{0}(x)$ a.e. in $\mathbb{R}^{N}$. By Fatou's lemma,

$$
\begin{aligned}
m & =\lim _{n \rightarrow \infty} I_{0}\left(u_{n}\right)=\lim _{n \rightarrow \infty} I_{0}\left(\widetilde{u}_{n}\right) \\
& =\liminf _{n \rightarrow \infty} \int \widehat{F}_{0}\left(x, \widetilde{u}_{n}\right) \\
& \geq \int \widehat{F}_{0}\left(x, u_{0}\right)=I_{0}\left(u_{0}\right) .
\end{aligned}
$$


Consequently $I_{0}\left(u_{0}\right)=m$ and therefore $u_{0} \neq 0$ is a ground state solution.

\section{ACKNowledgments}

This is part of the author?s Ph.D. thesis, written under the supervision of Dr. Marcelo F. Furtado at the University of Brasília. The author would like to express his sincere appreciation to his advisor for his guidance and advice throughout this research.

\section{REFERENCES}

[1] S. Alama and Y. Y. Li. On "multibump" bound states for certain semilinear elliptic equations. Indiana Univ. Math. J., 41 (1992), 983-1026.

[2] C. O. Alves, J. M. B. do Ó and O. H. Miyagaki. On perturbations of a class of a periodic $m$ laplacian equation with critical growth. Nonlinear analysis, theory, methods $\mathcal{E}$ applications, 45 (2001), 849-863.

[3] C. O. Alves, P. C. Carrião and O. H. Miyagaki. Nonlinear perturbations of a periodic elliptic problem with critical growth. Journal of mathematical analysis and applications, 260 (2001), 133-146.

[4] A. Bahri and Y. Li. On a min-max procedure for the existence of a positive solution for certain scalar field equations in $\mathbb{R}^{N}$. Revista Matematica Iberoamericana, 6 (1990), 1-15.

[5] H. Berestycki and P. L. Lions. Nonlinear scalar field equations, ii existence of infinitely many solutions. Archive for Rational Mechanics and Analysis, 82 (1983), 347-375.

[6] J. Chabrowski. Weak convergence methods for semilinear elliptic equations. (River Edge NJ: World Scientific Publishing Co., 1999).

[7] Y. Ding and C. Lee. Multiple solutions of schrödinger equations with indefinite linear part and super or asymptotically linear terms. Journal of Differential Equations, 222 (2006), 137-163.

[8] Y. Ding and A. Szulkin. Bound states for semilinear schrödinger equations with sign-changing potential. Calculus of Variations and Partial Differential Equations, 29 (2007), 397-419.

[9] H. F. Lins and E. A. B. Silva. Quasilinear asymptotically periodic elliptic equations with critical growth. Nonlinear Analysis: Theory, Methods $\&$ Applications, 71 (2009), 28902905.

[10] P. L. Lions. The concentration-compactness principle in the calculus of variations. the locally compact case, part 2. 1 (1984), 223-283.

[11] P. H. Rabinowitz. On a class of nonlinear schrödinger equations. Zeitschrift für Angewandte Mathematik und Physik (ZAMP), 43 (1992), 270-291.

[12] E. A. B. Silva and G. F. Vieira. Quasilinear asymptotically periodic schrödinger equations with critical growth. Calculus of Variations and Partial Differential Equations, 39 (2010), $1-33$.

[13] E. A. B. Silva and G. F. Vieira. Quasilinear asymptotically periodic schrödinger equations with subcritical growth. Nonlinear Analysis: Theory, Methods $\&$ Applications, 72 (2010), $2935-2949$.

[14] A. Szulkin and T. Weth. The method of nehari manifold. Handbook of nonconvex analysis and applications, (2010), 597-632.

[15] V. Coti Zelati and P. H. Rabinowitz. Homoclinic type solutions for a semilinear elliptic pde on $\mathbb{R}^{N}$. Communications on pure and applied mathematics, 45 (1992), 1217-1269. 